\title{
Das adäquate Niveau an Net Working Capital
}

Mit Supply Chain Finance lässt sich die Perspektive des Working Capital Managements auf die gesamte Supply Chain erweitern. Neuartige Lösungen ermöglichen nicht nur eine Verbesserung von Bilanzkennzahlen und Finanzierungskosten, sondern helfen Unternehmen auch, ein adäquates Niveau an Net Working Capital zu erreichen.

Philipp Wetzel, Anastasia Movcharenko 
Es gibt kaum ein Finanzthema im Unternehmen, das so viele unterschiedliche Funktionsbereiche tangiert wie das Nettoumlaufvermögen (Working Capital Management - WCM). Aus diesem Grund stellt die Steuerung des WCMs eine Daueraufgabe dar und bedarf einer langfristigen, strategischen Verankerung im Unternehmen. Wesentlich dabei ist, die Verhaltensweisen gegenüber Lieferanten und Kunden zur Erreichung der angestrebten unternehmerischen Ziele zu definieren. In der betrieblichen Praxis gilt es nicht nur, die internen WCM-Zielkonflikte zwischen den einzelnen Unternehmensbereichen, sondern insbesondere auch jene, die mit den Supply-Chain-Partnern bestehen, auszugleichen. Einseitige und unkooperative Working-Capital-Verbesserungen, wie beispielsweise die systematische Verlängerung oder Verkürzung von Zahlungszielen, gilt es zu vermeiden, da diese auf Kosten der vor- respektive nachgelagerten Supply-Chain-Partner erzielt werden.

Eine netzwerkbezogene Analyse des Cash-to-Cash-Zyklus (C2C Cycle) entlang der Supply Chain von Unternehmen im Swiss Performance Index (SPI) verdeutlicht im Zeitverlauf die Wichtigkeit eines kooperativen WCM-Ansatzes. Der C2C Cycle wird als Zeitraum definiert, der benötigt wird, um Zahlungen an den Zulieferer in Barzahlungen von Kunden umzuwandeln. Konkret weisen die Analyseergebnisse darauf hin, dass sich WCM-Maßnahmen von Schweizer SPI-Unternehmen in Form von Übertragungseffekten bei ihren Kunden und Lieferanten quantitativ auswirken (vergleiche Methodik, S. 29).

\section{„Die allgemeine Lehrbuchmeinung im Supply Chain Controlling, less is always more ist überholt."}

Im Jahr 2015 beispielsweise steigt der C2C Cycle der Top-5-Kunden der berücksichtigten SPI-Unternehmen um durchschnittlich 16 Prozent. Gleichzeitig verkürzte sich der C2C Cycle der vorgelagerten Supply-Chain-Partner um elf Prozent beziehungsweise zwei Prozent im Vergleich zum Vorjahr (vergleiche Abbildung 1). Diese Übertragungseffekte sind primär auf eine zunehmende Vernetzung der Märkte und eine hohe Lieferanten- und Kundenkonzentration zurückzuführen. So geht aus der WCM-Expertenbefragung hervor, dass 80 Prozent der Beschaffungskosten durch 14 Prozent der gesamten Lieferantenbasis generiert werden. Ein ähnliches Bild zeigt sich auf der Absatzseite: 80 Prozent des Umsatzes werden mit durchschnittlich 19 Prozent der gesamten Kundenbasis erzielt (vergleiche Hofmann/Wetzel 2018).

\section{Die optimale Balance finden}

Die WCM-Übertragungseffekte entlang der Supply Chain untermauern die Notwendigkeit einer ganzheitlichen Betrachtung des Nettoumlaufvermögens. Denn bei einer einseitigen Reduktion des Nettoumlaufvermögens erhöht sich im Gegenzug die relative Kapitalbindung bei Kunden und Lieferanten. Es besteht die Gefahr, dass Letztere hierdurch in Liquiditätsengpässe geraten und sich die langfristigen Geschäftsbeziehungen verschlechtern.

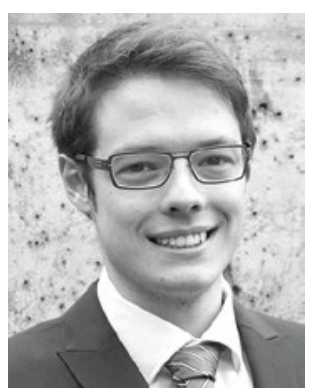

\section{Philipp Wetzel}

ist wissenschaftlicher Mitarbeiter am Institut für Supply Chain Management (ISCM-HSG) und Projekt-Manager des Supply Chain Finance Labs (SCF-Lab) an der Universität St. Gallen (HSG). E-Mail:philipp.wetzel@unisg.ch

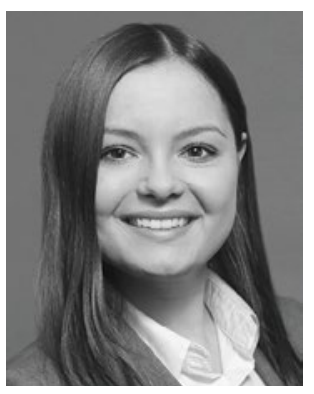

\section{Anastasia Movcharenko}

ist Projektmitarbeiterin am Institut für Supply Chain Management an der Universität St. Gallen (ISCM-HSG).

E-Mail: anastasia.movcharenko@student. unisg.ch 
Vergleicht man die Unternehmens-Performance mit den C2C Cycles von Schweizer SPI-Unternehmen, so zeigt sich, dass jene Unternehmen, die einen leicht positiven C2C Cycle aufweisen, die also das Kapital für die entsprechenden Transaktionen für nur kurze Zeit gebunden haben, ihr Working Capital am effizientesten einsetzen. Die Resultate deuten darauf hin, dass zwischen der Unternehmens-Performance, approximiert mit dem Return on Capital Employed (ROCE), und dem C2C Cycle (in Tagen) ein invertierter U-förmiger Zusammenhang besteht. Die Analyse legt demnach nahe, dass ein optimales Niveau an Nettoumlaufvermögen existiert, welches positive und negative Effekte miteinander ausbalanciert und gleichzeitig die UnternehmensPerformance maximiert (vergleiche Hofmann et al. 2017). Wichtig dabei ist zu verstehen, dass das unternehmensspezifische optimale Niveau an Nettoumlaufvermögen von verschiedenen Treibern und Einflussfaktoren, wie beispielsweise den Branchen-Charakteristika, abhängt (vergleiche Abbildung 2).

\section{„Das unternehmensspezifische optimale Niveau an Nettoumlaufvermögen hängt von verschiedenen Treibern und Einflussfaktoren ab."}

Des Weiteren impliziert die Analyse, dass eine Reduktion des Nettoumlaufvermögens nur bis zu einem gewissen Niveau einen positiven Effekt auf die Unternehmens-Performance ausübt. Wird die „Schwelle“ des optimalen Niveaus unterschritten (wird der C2C Cycle also zu stark reduziert), scheint sich eine zusätzliche Reduktion des Working Capitals negativ auf die Performance auszuwirken. Diese Erkenntnis folgt dem Gedanken der goldenen

\section{Abb. 1 WCM-Übertragungseffekte entlang der Supply Chain}

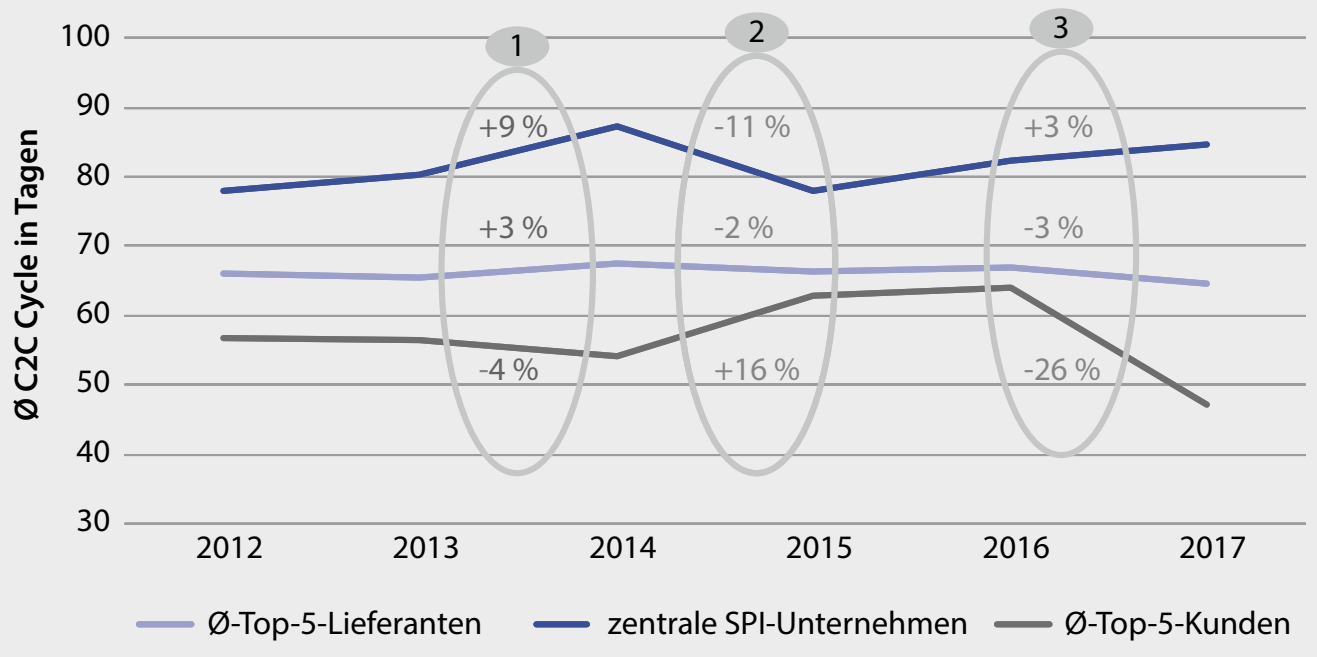

Quelle: eigene Darstellung 
Bilanzregel, wonach die Kapitalbindungsdauer und die Finanzierungsdauer aufeinander abzustimmen sind (fristenkongruente Finanzierung).

\section{Optimieren mit Supply Chain Finance}

Vor dem Hintergrund des invertierten U-förmigen Zusammenhangs kann die Optimierung des Nettoumlaufvermögens nur erfolgreich sein, wenn die Folgen von unternehmerischen Entscheidungen sowohl für das eigene Unternehmen als auch für die Supply-Chain-Partner adäquat berücksichtigt werden (Hofmann/Kotzab 2010). Ausgehend von dieser Perspektive hat Supply Chain Finance (SCF) die unternehmensübergreifende Optimierung des Nettoumlaufvermögens sowie die Integration der Finanzprozesse zwischen Kunden, Lieferanten und externen Dienstleistern zum Ziel. SCF erfüllt damit den Anspruch, die unterschiedlichen Interessen und Zielsetzungen im WCM entlang des unternehmensübergreifenden Wertschöpfungsprozesses auszugleichen und für die beteiligten Akteure zu verbessern (vergleiche Templar et al. 2016).

Vor diesem Hintergrund stellt SCF für Unternehmen ein flexibles Instrument dar, das ganz gezielt zur Anvisierung des performancemaximierenden

\section{Entlang der Supply Chain von Unternehmen zeigen sich erhebliche WCM- Übertragungseffekte.}

Abb. 2 Zusammenhang zwischen C2C Cycle und Unternehmens-Performance

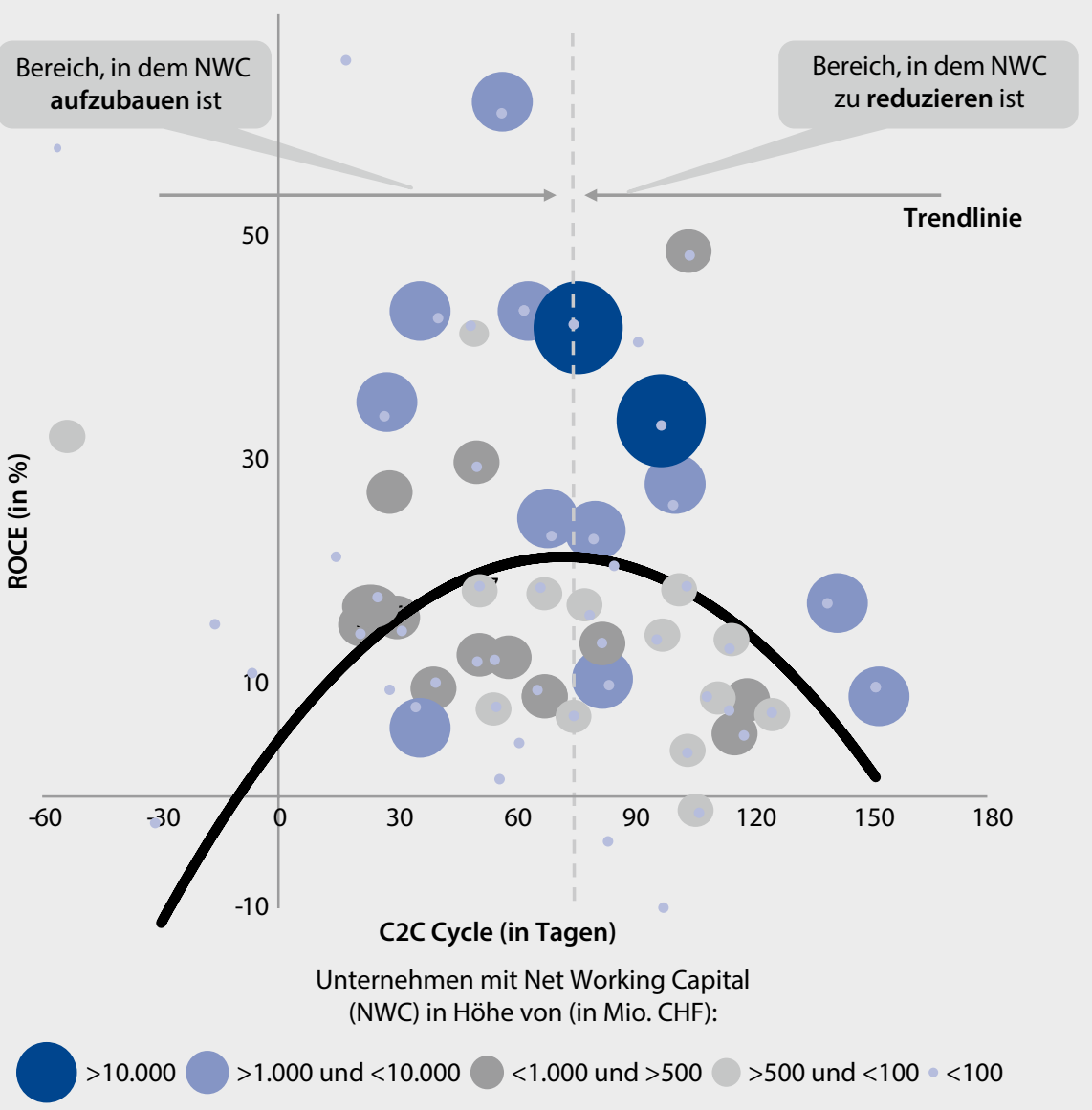

Quelle: eigene Darstellung 


\section{Zusammenfassung}

- Es reicht nicht, nur die WorkingCapital-Management-Zielkonflikte zwischen den einzelnen Unternehmensbereichen auszugleichen. Auch jene, die mit externen Supply-Chain-Partnern bestehen, müssen gelöst werden.

- Net Working Capital hat dann ein performancemaximierendes Niveau, wenn es positive und negative Effekte miteinander ausbalanciert.

- Supply Chain Finance hat die unternehmensübergreifende Optimierung des Nettoumlaufvermögens sowie die Integration der Finanzprozesse zwischen Kunden, Lieferanten und externen Dienstleistern zum Ziel.
Niveaus an Net Working Capital eingesetzt werden kann. Letzteres Niveau bestimmt dabei jene Mittel, die erforderlich sind, um den operativen Zyklus eines Unternehmens zu finanzieren und gleichzeitig die strategischen Ziele gegenüber Kunden und Lieferanten bestmöglich zu erfüllen. Das adäquate Niveau wird erreicht, wenn die Differenz zwischen dem entstehenden Nutzen (= positive Effekte) und den anfallenden Kosten (= negative Effekte) der Kapitalbindung bestmöglich ausbalanciert wird.

\section{„Eine Reduktion des Nettoumlaufvermögens hat nur bis zu einem gewissen Niveau einen positiven Effekt auf die Unternehmens-Performance."}

Die Studienergebnisse zeigen, dass derzeit eine Reihe von innovativen SCF-Lösungen auf dem Markt existiert, die sich verschiedenster digitaler Möglichkeiten bedient, um diese Integrationsleistung zu erfüllen (vergleiche Abbildung 3). Daraus ergeben sich nicht nur weitreichende Konsequenzen, sondern insbesondere auch neue Möglichkeiten für ein ganzheitliches WCM in der Supply Chain (Wetzel et al. 2018). Besonders für die WCMBezugspunkte Debitoren und Kreditoren existiert auf dem Markt bereits eine Vielzahl an technologisch ausgereiften SCF-Lösungen. Im Bestandsbereich sind die Lösungsansätze aktuell noch weniger ausgereift. Die Umfrageergebnisse belegen jedoch, dass gerade Bestandsfinanzierungslösungen für Unternehmen häufig die größten Potenziale bieten. Allgemein ist der Trend erkennbar, dass vermehrt Lösungen entwickelt werden, die sich durch eine hochgradige Vernetzungsfähigkeit, Modularisierung und Technologiedurchdringung auszeichnen. Ein Beispiel dafür stellen bankenunabhängige SCF-Plattformen wie CRX Markets dar, die Investoren und Unternehmen mittels nahtloser IT-Integration zusammenbringen. Gleichzeitig findet eine Automatisierung der unternehmensübergreifenden Abwicklungsprozesse zwischen Abnehmer und Lieferanten statt, die eine erhebliche Vereinfachung der Zusammenarbeit bei sehr geringem Integrationsaufwand und

\section{Abb. 3 Physische und finanzielle Integration der Up- und Downstream-Supply-Chain-Partner}

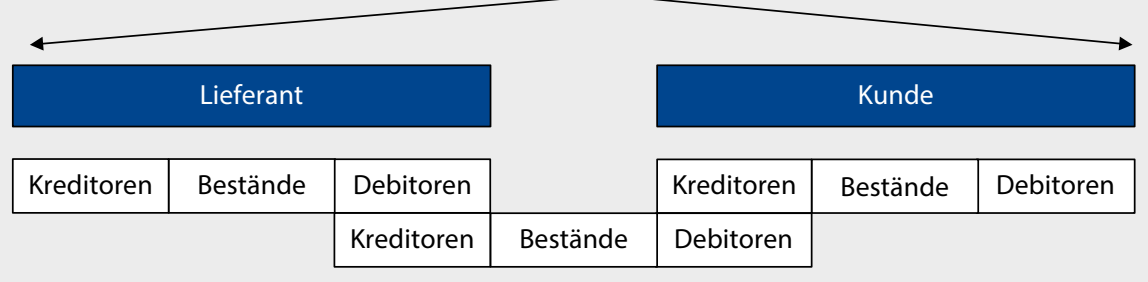


hoher Datensicherheit ermöglicht (vergleiche Abbildung 4). Daraus ergeben sich für Unternehmen nicht nur weitreichende Konsequenzen, sondern insbesondere auch neue Möglichkeiten, um ihr performancemaximierendes Niveau an Net Working Capital zu erreichen.

\section{Supply Chain Finance bei Nestlé}

Komplexe Supply-Chain-Strukturen von internationalen Großkonzernen erfordern ein effizientes und agiles Lieferanten-Management. In diesem $\mathrm{Zu}$ sammenhang stand Nestlé vor der Herausforderung, seine Beschaffungsfunktionen in den globalen Märkten zu zentralisieren sowie die finanzielle Stabilität seiner strategischen Lieferanten zu sichern.

Mit der SCF-Plattform-Lösung von CRX konnte Nestlé einen integrierten Ansatz finden, der eine homogene, flexible und leicht skalierbare Finanzierungslösung für unterschiedliche Lieferantengruppen bietet. Durch die einfache und nahtlose Integration der CRX-Lösung in das ERP-System von Nestlé (via Add-on) konnten die bestehenden Beschaffungsprozesse weitestgehend erhalten bleiben und die nachgelagerten Prozesse signifikant verbessert werden. Der Prozess der Lieferantenfinanzierung läuft dabei wie folgt ab: Nach Warenlieferung und der Rechnungsstellung durch den Lieferanten prüft der Abnehmer Nestlé die Rechnungsdaten und lädt diese auf die CRXPlattform hoch. Im Anschluss werden die verifizierten Forderungen gebündelt und in ein handelbares Finanzprodukt verbrieft. Die ausgewählten Partnerbanken und professionelle Investoren können nun die durch Nestlé

\author{
Wird das optimale Niveau \\ des Working Capitals \\ unterschritten, wirkt sich \\ dies negativ auf die \\ Performance aus.
}

\section{Abb. 4 Ausgewählte SCF-Lösungen für zentrale Unternehmen und deren Potenziale}

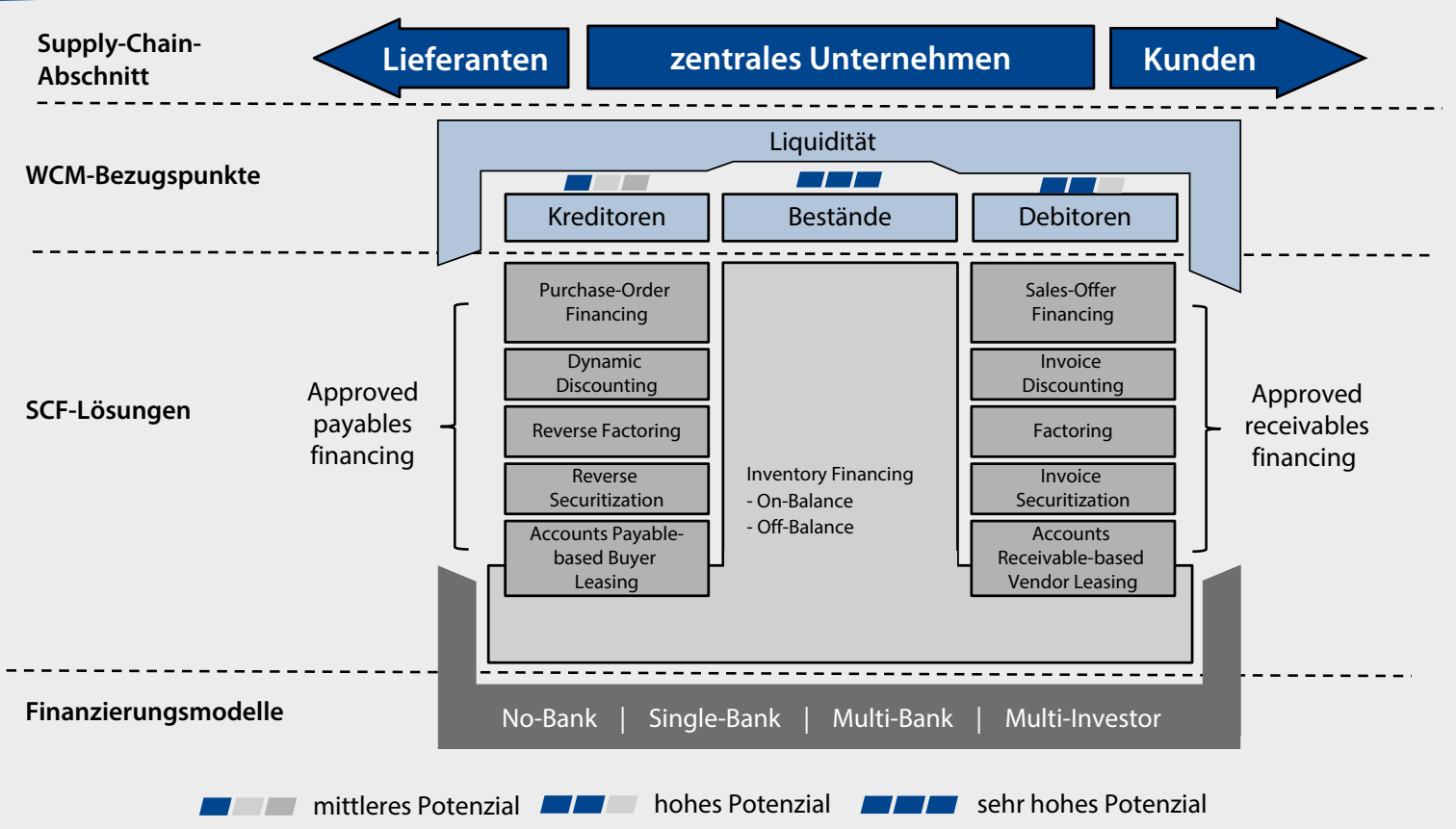

Quelle: eigene Darstellung 


\section{Supply Chain Finance hilft}

Unternehmen, das optimale Niveau an Net Working Capital zu erreichen. bestätigten Lieferantenforderungen über die integrierte Auktionsplattform ersteigern. Die Zahlung der erfolgreich verkauften Forderungen erfolgt in der Regel innerhalb von wenigen Tagen auf das Konto der Lieferanten nach Abschluss des Settlement-Prozesses. Gleichzeitig profitiert der Abnehmer Nestlé von verlängerten Zahlungszielen und bezahlt den Normalbetrag der Lieferantenrechnung zum vereinbarten Fälligkeitstermin (vergleiche Abbildung 5). CRX bietet Nestlé und seinen Lieferanten damit über mehrere Kanäle Zugang zu attraktiven Working-Capital-Finanzierungen. Die PlattformLösung unterscheidet sich insofern von klassischen Finanzierungslösungen, als dass Lieferanten durch die breite Investorenbasis und die gute Bonität von Nestlé eine deutlich günstigere und flexiblere Finanzierung ihres Nettoumlaufvermögens erhalten.

\section{Handlungsempfehlungen für Finanzverantwortliche}

Mit SCF beziehen Unternehmen alle Partner ihrer Wertschöpfungskette in ihr WCM mit ein und verschaffen sich so Wettbewerbsvorteile. In der Breite nutzen insbesondere mittelständische Unternehmen kooperative SCFInstrumente jedoch noch immer zurückhaltend. Und dies, obwohl der Markt derzeit rasant wächst und innovative Lösungen zur Verfügung stehen, die Möglichkeiten für ein ganzheitliches WCM in der Supply Chain bieten. Daraus lassen sich für Geschäftsführer und Finanzverantwortliche insbesondere zwei Handlungsempfehlungen ableiten:

\section{Abb. 5 Flexible, automatisierte und transparente Lieferantenfinanzierung bei Nestlé}

1

Waren und
Rechnung

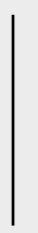

Lieferanten

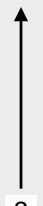

8

Zahlung an die

Lieferanten nach

Abschluss des

Settlement-Prozesses
2

Prüfung und

Bestätigung

Rechnungen

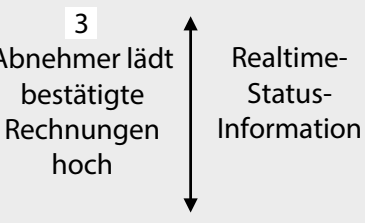

CRX Markets

5

1. Auktionsankündigung

2. Gebote

3. Bestätigung

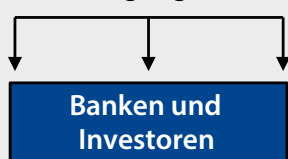

10

Rückzahlung

der Note bei

SPV erwirbt die

Forderung und

begibt die Note

nach erfolgreicher

Auktion

9

Abnehmer zahlt

bei Fälligkeit

Normalbetrag

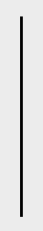

SPV und Clearing

Fälligkeit 
- Bei einem kooperativen WCM-Ansatz geben stets die strategischen Ziele den Ton an. Möchte sich etwa ein Unternehmen von den Mitbewerbern abheben, indem es sich bei seinen wichtigsten Lieferanten als „Preferred Customer" positioniert, ist es sinnvoll, offene Rechnungen rasch zu bezahlen. Um dieses Ziel ohne Verschlechterung der eigenen Liquiditätssituation erfüllen zu können, eignet sich etwa das SCF-Instrument „Reverse Factoring", mit dem die Lieferanten ihr Geld früher erhalten und sich der eigene DPO (Days Payables Outstanding) beispielsweise auf 90 Tage verlängern lässt. So entsteht eine klassische Win-win-Situation, wie sie für den kooperativen WCM-Ansatz typisch ist.

- Möchte sich ein Unternehmen gleichzeitig auf der Absatzseite als „Preferred Supplier“" positionieren, empfiehlt sich etwa das SCF-Instrument „Stilles Factoring", mit dem das Unternehmen die Kundenforderungen an einen Finanzdienstleister abtritt. Das stille Factoring eignet sich beispielsweise, wenn Kunden potenziell sensibel auf Forderungsverkäufe reagieren oder wenn ein Unternehmen vermeiden möchte, dass seine Kunden annehmen, es sei in finanzielle Schwierigkeiten geraten. Denn beim stillen Factoring erfahren die Kunden nicht, dass zur Finanzierung der Forderungen ein externer Finanzdienstleister beigezogen wurde.

\section{Schlussbetrachtung}

Durch seinen Querschnittscharakter verlangt ein ganzheitliches WCM, dass Unternehmen nicht nur die internen WCM-Zielkonflikte zwischen den einzelnen Unternehmensbereichen, sondern insbesondere auch jene ausgleichen, die mit den Supply-Chain-Partnern, also Kunden und Lieferanten, bestehen. Übereinstimmend mit der neoklassischen Theorie, zeigt die Analyse bei Schweizer SPI-Unternehmen, dass eine Reduktion des Working Capitals nur bis zu einem bestimmten Niveau einen positiven Effekt auf die Unternehmens-Performance hat. Große Bestände erhöhen beispielsweise zwar das gebundene Kapital, stellen aber auch die Kundenzufriedenheit sicher. Ähnliche Spannungsfelder gilt es, auch im Debitoren-, Kreditoren- und Liquiditäts-Management aufzulösen. Die allgemeine Lehrbuchmeinung im Supply Chain Controlling „less is always more“ ist überholt.

\section{„Insbesondere mittelständische Unternehmen nutzen kooperative SCF-Instrumente noch immer zurückhaltend, obwohl innovative Lösungen zur Verfügung stehen."}

Für Unternehmen besteht im WCM folglich eine zentrale Aufgabe darin, das Net Working Capital und dessen Zielkonflikte gemeinsam mit allen anderen Partnern in der Supply Chain zu optimieren. SCF als WCM-Instrument erweitert die Perspektive des WCMs auf die gesamte Supply Chain und bietet Unternehmen diese Möglichkeit. Das versetzt Unternehmen in die

\section{Methodik}

Diesem Beitrag liegen zwei verschiedene Datenquellen zugrunde. Zum einen basieren die Auswertungen auf einer Stichprobe von insgesamt 114 Schweizer Unternehmen aus elf verschiedenen Branchen. Die Stichprobe setzt sich zu 30 Prozent aus Großunternehmen (>250 Mitarbeitende) und zu 70 Prozent aus kleinen und mittelständischen Unternehmen (KMU) zusammen. Im Rahmen der Expertenumfrage wurden sowohl qualitative Einschätzungen als auch quantitative Angaben abgefragt. Alle angegebenen Informationen und Daten wurden eingehend geprüft, validiert und systematisch aufbereitet. 61 Prozent der 114 Fragebögen wurden durch CFOs oder CEOs ausgefüllt. Zum anderen wurde für die netzwerkbezogene Analyse auf öffentlich zugängliche Daten aus verschiedenen Wirtschafts- und Finanzdatenbanken (insbesondere Bloomberg und Thomson Reuters Eikon) zurückgegriffen. 
Lage, eine ganzheitliche WCM-Strategie umzusetzen, bei der sie sich durch überdurchschnittliche Produkt- oder Service-Leistungen für ihre SupplyChain-Partner von anderen Mitbewerbern abheben können, so zum Beispiel durch die rasche Zahlung von Rechnungen. Von dieser Warte aus betrachtet, stellt WCM nicht nur eine operative Management-Aufgabe dar, sondern hilft Unternehmen dabei, langfristige Unternehmensziele wie die Erhöhung des finanziellen Spielraums, die Optimierung der Liquidität, die Senkung von Finanzierungskosten sowie die Verbesserung der operativen Marge und Rentabilität zu erreichen.

\section{Literatur}

Hofmann, E./Wetzel, P. (2018): Working Capital Management Studie 2018 - Supply Chain Finance. Supply Chain Finance-Lab (SCF-Lab) an der Universität St. Gallen, Bern.

Hofmann, E./Wetzel, P./Schneider, C. (2017): Working Capital Management bei Eisenbahnverkehrsunternehmen, in: Controller Magazin, 42 (5), S. 4-13.

Hofmann, E./Kotzab, H. (2010): A Supply Chain-Oriented Approach of Working Capital Management, in: Journal of Business Logistics, 21 (2), S. 305-330.

Templar, S./Hofmann, E./Findlay, C. (2016): Financing the End-to-End Supply Chain: A Reference Guide to Supply Chain Finance, London.

\section{Springer Professional.de}

Spiecker-Lampe, C. (2018): Nachhaltige Optimierung von Kapitalkosten im Mittelstand mit Supply Chain Finance, in: Dovbischuk, I./Siestrup, G./Tuma, A. (Hrsg.): Nachhaltige Impulse für Produktion und Logistikmanagement, S. 177-187. www.springerprofessional.de/link/15568182

Hofmann, E./Strewe, U. M./Bosia, N. (2018): Supply Chain Finance and Blockchain Technology. The Case of Reverse Securitisation, Cham (Schweiz). www.springerprofessional.de/link/13350776

Zhao, L./Huchzermeier, A. (2018): Supply Chain Finance. Integrating Operations and Finance in Global Supply Chains, Cham (Schweiz).

www.springerprofessional.de/link/15734148 